\title{
Radiative improvement of the lattice NRQCD action with application to hyperfine splittings
}

\author{
T.C. Hammant ${ }^{a}$, A.G. Hart ${ }^{b}$, G.M. von Hippel ${ }^{* c}$, R.R. Horgan ${ }^{a}$, C.J. Monahan ${ }^{d}$ \\ ${ }^{a}$ Department of Applied Mathematics and Theoretical Physics, University of Cambridge, \\ Cambridge CB3 OWA, UK \\ ${ }^{b}$ Cray Exascale Research Initiative Europe, JCMB, King's Buildings, Edinburgh EH9 3JZ, UK \\ ${ }^{c}$ Institut für Kernphysik, Johannes Gutenberg-Universität Mainz, 55099 Mainz, Germany \\ ${ }^{d}$ Department of Physics, College of William and Mary, VA 23187-8795, USA \\ Email: hippelekph.uni-mainz.de
}

\begin{abstract}
Recent results for the radiative improvement of the lattice NRQCD action, specifically for the $\sigma \cdot B$ term, the Darwin term, and the non-derivative four-fermion terms, are presented. The corrections to the spin-dependent terms are found to have a significant impact on the value of the hyperfine splitting, both for bottomonia and heavy-light mesons, pushing the theoretical prediction towards the experimental value. The spin-independent terms are affected by both lattice artifacts and severe IR divergences, rendering their evaluation complicated.
\end{abstract}

Xth Quark Confinement and the Hadron Spectrum

8-12 October 2012

TUM Campus Garching, Munich, Germany

\footnotetext{
* Speaker.
} 


\section{Introduction}

Non-Relativistic QCD (NRQCD) [1] on the lattice has been used for a successful description of hadrons containing heavy quarks. The lattice NRQCD action used by the HPQCD collaboration is given by

$$
S=\sum_{\vec{x}, \tau} \psi^{\dagger}(\vec{x}, \tau)[\psi(\vec{x}, \tau)-K(\tau) \psi(\vec{x}, \tau)]
$$

with the kernel

$$
K(\tau)=\left(1-\frac{\left.\delta H\right|_{\tau}}{2}\right)\left(1-\frac{\left.H_{0}\right|_{\tau}}{2 n}\right)^{n} U_{4}^{\dagger}(\tau-1)\left(1-\frac{\left.H_{0}\right|_{\tau-1}}{2 n}\right)^{2}\left(1-\frac{\left.\delta H\right|_{\tau-1}}{2}\right)
$$

where

$$
\begin{gathered}
H_{0}=\frac{\Delta^{(2)}}{2 M_{0}}, \quad \delta H=-c_{1} \frac{\left(\Delta^{(2)}\right)^{2}}{8 M_{0}^{3}}+c_{2} \frac{i g}{8 M_{0}^{2}}\left(\vec{\Delta}^{ \pm} \cdot \vec{E}-\vec{E} \cdot \vec{\Delta}^{ \pm}\right) \\
-c_{3} \frac{g}{8 M_{0}^{2}} \vec{\sigma} \cdot\left(\vec{\Delta}^{ \pm} \times \vec{E}-\vec{E} \times \vec{\Delta}^{ \pm}\right)-c_{4} \frac{g}{2 M_{0}} \vec{\sigma} \cdot \vec{B}+c_{5} a^{2} \frac{\Delta^{(4)}}{24 M_{0}}+c_{6} a \frac{\left(\Delta^{(2)}\right)^{2}}{16 n M_{0}^{2}},
\end{gathered}
$$

with the operators normalized so as to put the tree-level values of the matching coefficient equal to one, $c_{i}=1+\mathscr{O}\left(\alpha_{s}\right)$, and $n \geq 3 /\left(M_{0} a\right)$ is a stability parameter.

Until recently, the higher-order corrections to the coefficients in the action were not known. We have now demonstrated [2] that the combination of the radiative improvement of the coefficient $c_{4}$ of the chromomagnetic moment operator and the inclusion of spin-dependent four-fermion operators into the action leads to a significant shift in the value of the bottomonium hyperfine splitting, putting the theoretical prediction into agreement with experiment. Here we present an update on the improvement of these spin-dependent operators, as well as first results for the radiative improvement of the Darwin term and the leading spin-independent four-fermion operators (cf. also [3]).

\section{NRQCD/QCD Matching Using Background Field Gauge}

To extend the matching to the one-loop level, we require some suitably chosen set of renormalized one-loop S-matrix elements to agree between QCD and NRQCD. Since the terms in $\delta H$ are bilinear in the quark field, quark scattering off a background field can be used, leading to the condition that QCD and NRQCD give the same effective potential after non-relativistic reduction:

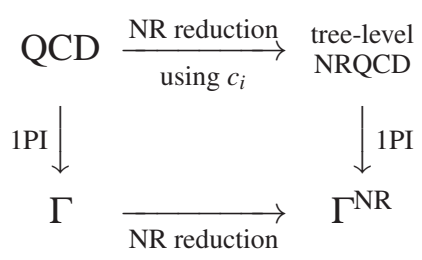

The effective potential in the presence of a classical background field $B_{\mu}$ is defined by

$$
\mathrm{e}^{-\Gamma\left[B_{\mu}\right]}=\int_{1 \mathrm{PI}} \mathscr{D} q_{\mu} \mathrm{e}^{-S\left[B_{\mu}+g q_{\mu}\right]},
$$

where the path integral over the quantum fluctuations $q_{\mu}$ is defined only in perturbation theory restricted to 1 PI diagrams. 


\subsection{Calculating in background field gauge}

The BRST invariance of the classical action $S$ guarantees that all $D \leq 4$ operators appearing in the effective action $\Gamma$ are gauge covariant, implying in particular the renormalizability of QCD. The $D>4$ operators that will appear in $\Gamma$ are, however, not necessarily gauge covariant. Since NRQCD is an effective theory, its classical action $S$ contains $D>4$ operators. Gauge covariance can be imposed at tree level. At the level of loop corrections, gauge covariance must also be retained to avoid serious complications: although the appearance of gauge-noncovariant operators as such would not invalidate the theory, having to keep track of their gauge dependence in subsequent calculations would be a significant annoyance.

As has been shown long ago, [4], the gauge covariance of the effective action can be assured by using background field gauge (BFG), which is given by the gauge fixing function

$$
f(A)=D_{\mu}^{B} q^{\mu}=\left(\partial_{\mu}+i B_{\mu}\right) q^{\mu},
$$

so that not only the propagator, but also the vertices with exactly two quantum gluons depend on the gauge parameter. On the lattice, the background and quantum fields are defined by decomposing the gauge link into the ordered product

$$
U_{\mu}(x)=\mathrm{e}^{g_{0} q_{\mu}\left(x+\frac{1}{2} \hat{\mu}\right)} \mathrm{e}^{B_{\mu}\left(x+\frac{1}{2} \hat{\mu}\right)} .
$$

Since the quantum field occurs to the left of the background field, the Feynman rules will depend not only on the number of background and quantum fields entering a vertex, but will also contain different terms from different orderings (e.g. $B q q, q B q, q q B$ ) that contribute to the same vertex. The discretized gauge fixing function

$$
f(A)=D_{\mu}^{B} q_{\mu}(x)=\left[q_{\mu}(x)-\mathrm{e}^{-B_{\mu}\left(x-\frac{\hat{\mu}}{2}\right)} q_{\mu}(x-\hat{\mu}) \mathrm{e}^{B_{\mu}\left(x-\frac{\hat{\mu}}{2}\right)}\right]
$$

again affects all vertices with exactly two quantum gluons (of which there is an infinite sequence on the lattice). The HiPPY/HPsrc packages for automated lattice perturbation theory [5] have been extended to support the automated derivation of the Feynman rules also for BFG calculation.

Lattice gauge theories in BFG are known to be renormalizable [6]. An important feature of BFG which we will exploit in the following is that it implies a set of QED-like Ward identities and finite counterterms; we can therefore compute both the lattice and the continuum diagrams numerically, and we do not need to calculate the gauge field renormalization. We benefit from this especially in that it allows us to easily check the gauge-parameter independence of our results for $c_{4}$ and $c_{2}$ (which have to be gauge-parameter independent, since they could also be defined nonperturbatively in terms of physical mass splittings). The non-trivial gauge-parameter independence of the results is an important check on their correctness.

\subsection{Matching bilinears}

The effective action for continuum QCD contains terms of the form

$$
\Gamma[\Psi, \bar{\Psi}, A]=\bar{\Psi} F_{1}\left(q^{2}\right) \not \supset \Psi+\bar{\Psi} \frac{F_{2}\left(q^{2}\right) \sigma^{\mu v} F_{\mu v}}{2 M} \Psi+\ldots
$$




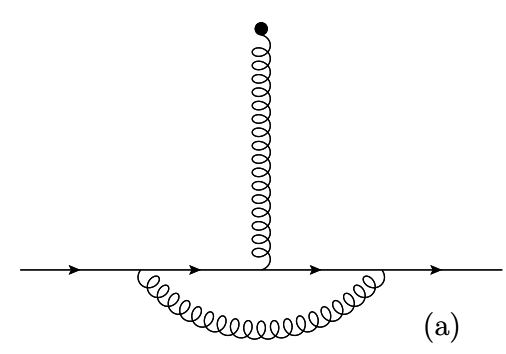

(a)

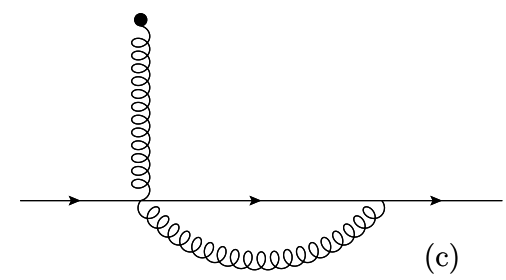

(c)

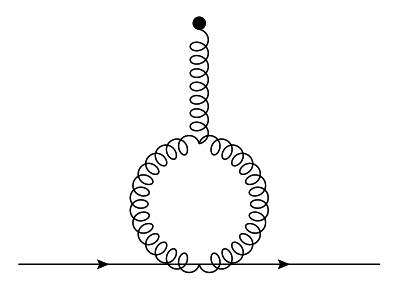

(e)

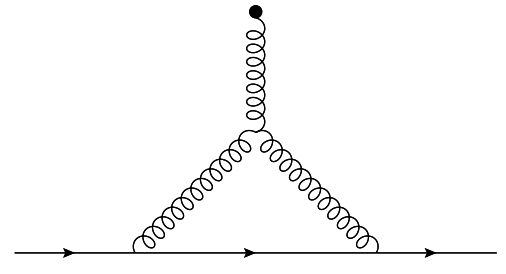

(b)

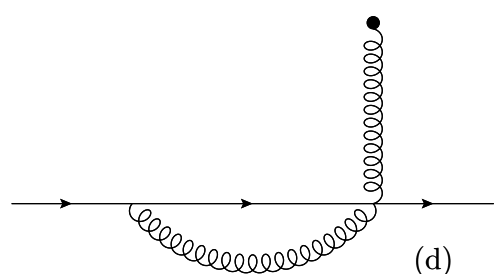

(d)

Figure 1: The one-particle irreducible diagrams entering the matching of the $\sigma \cdot B$ and Darwin terms in NRQCD at the one-loop level. On the QCD side, only diagrams (a) and (b) need to be computed, while in NRQCD all diagrams contribute; note that diagrams (c)-(f) contain not only lattice artifacts, but also physical contributions from higher orders in $1 / \mathrm{m}$.

which after renormalization and non-relativistic reduction give a $\sigma \cdot B$ term of

$$
(1+\underbrace{F_{2}(0) F_{1}(0)^{-1}}_{=b_{\sigma}}) \psi_{R}^{\dagger} \frac{\vec{\sigma} \cdot \vec{B}}{2 M_{R}} \psi_{R}
$$

and a Darwin term of

$$
(1-\underbrace{8 M_{R}^{2} F_{1}^{\prime}(0)+2 F_{2}(0)}_{=b_{D}}) \psi_{R}^{\dagger}\left(-\frac{g q^{2} A_{0}}{8 M_{R}^{2}}\right) \psi_{R} .
$$

A fairly straightforward calculation yields

$$
b_{\sigma}^{(1)}=\left(\frac{3}{2 \pi} \log \frac{\mu}{M}+\frac{13}{6 \pi}\right) \alpha \quad b_{D}^{(1)}=\left(-\frac{M^{2}}{\pi \mu^{2}}-\frac{7 M}{4 \mu}-\frac{1}{\pi}-\frac{50}{9 \pi} \log \frac{\mu}{M}\right) \alpha
$$

for the coefficients of these operators.

On the NRQCD side, the effective action contains the terms

$$
\Gamma^{\mathrm{NR}}\left[\psi, \psi^{\dagger}, A\right]=c_{4} Z_{\sigma}^{\mathrm{NR}} \psi^{\dagger} \frac{i \sigma \cdot(q \wedge A)}{2 M} \psi-c_{2} Z_{D}^{\mathrm{NR}} \psi^{\dagger} \frac{g q^{2} A_{0}}{8 M^{2}} \psi+\ldots
$$


which after renormalization give

$$
\Gamma^{\mathrm{NR}}\left[\psi_{R}, \psi_{R}^{\dagger}, A\right]=c_{4} Z_{\sigma}^{\mathrm{NR}} Z_{2}^{\mathrm{NR}} Z_{m}^{\mathrm{NR}} \psi_{R}^{\dagger} \frac{i \sigma \cdot(q \wedge A)}{2 M_{R}} \psi_{R}-c_{2} Z_{D}^{\mathrm{NR}} Z_{2}^{\mathrm{NR}}\left(Z_{m}^{\mathrm{NR}}\right)^{2} \psi_{R}^{\dagger} \frac{g q^{2} A_{0}}{8 M_{R}^{2}} \psi_{R}+\ldots
$$

Equating the corresponding terms in $\Gamma^{\mathrm{NR}}$ gives the matching conditions

$$
\begin{aligned}
c_{4} Z_{\sigma}^{\mathrm{NR}} Z_{2}^{\mathrm{NR}} Z_{m}^{\mathrm{NR}} & =1+b_{\sigma} \\
c_{2} Z_{D}^{\mathrm{NR}} Z_{2}^{\mathrm{NR}}\left(Z_{m}^{\mathrm{NR}}\right)^{2} & =1+b_{D}
\end{aligned}
$$

confirming $c_{i}^{(0)}=1$ at tree-level, and yielding the one-loop coefficients

$$
\begin{gathered}
c_{2}^{(1)}=b_{D}^{(1)}-\delta Z_{D}^{\mathrm{NR},(1)}-\delta Z_{2}^{\mathrm{NR},(1)}-2 \delta Z_{m}^{\mathrm{NR},(1)}, \\
c_{4}^{(1)}=b_{\sigma}^{(1)}-\delta Z_{\sigma}^{\mathrm{NR},(1)}-\delta Z_{2}^{\mathrm{NR},(1)}-\delta Z_{m}^{\mathrm{NR},(1)},
\end{gathered}
$$

where we have split the renormalization constants as $Z=1+\delta Z$.

In lattice NRQCD, $\delta Z_{\sigma, D, m}^{\mathrm{NR},(1)}$ receive contributions not only from the Feynman diagrams shown, but also from the mean-field improvement $U \mapsto U / u_{0}$ of the action, whereby the link variables are divided by their mean-field value to reduce higher-order corrections, and we take these "tadpole" contributions into account explicitly.

\subsection{Matching four-fermion operators}

Beyond tree level, the NRQCD action will also contain four-fermion terms

$$
\begin{aligned}
\mathscr{L}_{4 f}= & d_{1} \frac{\alpha_{s}}{M^{2}}\left(\psi^{\dagger} \chi^{*}\right)\left(\chi^{t} \psi\right)+d_{2} \frac{\alpha_{s}}{M^{2}}\left(\psi^{\dagger} \sigma \chi^{*}\right)\left(\chi^{t} \sigma \psi\right) \\
& +d_{3} \frac{\alpha_{s}}{M^{2}}\left(\psi^{\dagger} t^{a} \chi^{*}\right)\left(\chi^{t} t^{a} \psi\right)+d_{4} \frac{\alpha_{s}}{M^{2}}\left(\psi^{\dagger} \sigma t^{a} \chi^{*}\right)\left(\chi^{t} \sigma t^{a} \psi\right) \\
= & a_{1} \frac{g^{2}}{M^{2}}\left(\chi^{\dagger} \chi\right)\left(\psi^{\dagger} \psi\right)+a_{8} \frac{g^{2}}{M^{2}}\left(\chi^{\dagger} t_{a}^{t} \chi\right)\left(\psi^{\dagger} t_{a} \psi\right) \\
& +b_{1} \frac{g^{2}}{M^{2}}\left(\chi^{\dagger} \sigma^{*} \chi\right)\left(\psi^{\dagger} \sigma \psi\right)+b_{8} \frac{g^{2}}{M^{2}}\left(\chi^{\dagger} \sigma^{*} t_{a}^{t} \chi\right)\left(\psi^{\dagger} \sigma t_{a} \psi\right)
\end{aligned}
$$

where the coefficients $d_{i}$ that are linear combinations of the coefficients $a_{i}, b_{i}$ that can be determined by Fierz transformations.

The coefficients $a_{i}, b_{i}$ are most easily computable from the box diagrams of fig. 2. In addition to these, the coefficients $d_{i}$ receive contributions accounting for the fact that $Q \bar{Q}$ annihilation is possible in QCD, but not in NRQCD. For the purposes of determining the spin-dependent part, the relevant contribution is

$$
d_{1}^{\text {ann }}=-\frac{2 \alpha_{s}}{9 M^{2}}(2-2 \log 2) .
$$

\section{Divergences and artifacts}

While the final results for the matching coefficients must necessarily be IR-finite, the Feynman diagrams that need to be calculated are IR divergent in both QCD and NRQCD. We use a gluon mass $\mu$ to regulate these divergences. 


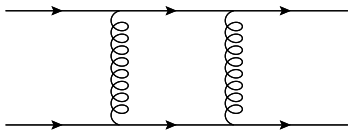

(a)

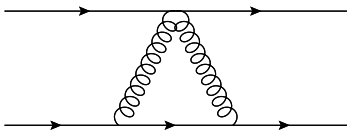

(c)

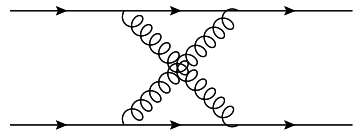

(b)

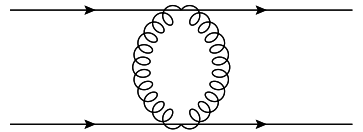

(d)

Figure 2: The one-particle irreducible diagrams entering the matching of the four-fermion interactions in NRQCD. In QCD, only the box and cross-box diagrams contribute, while in NRQCD all diagrams must be computed.

We note that the use of a gluon mass does not ruin the gauge-covariance of our results, as we could also have introduced the gluon mass through inducing the spontaneous symmetry breaking $S U(3) \rightarrow U(1)_{3} \times U(1)_{8}$, which for the quark bilinears gives precisely the diagrams we are calculating for the form factors of the (massless) $U(1)_{8}$ gluon when working in a renormalizable $R_{\xi}$ gauge. At higher loop orders there would be additional diagrams involving the remaining massless scalar fields; moreover, the existence of these fields means that in the limit $\mu \rightarrow 0$, QCD would not, in general, be recovered.

Another way to justify the use of a gluon mass is to note that at the one-loop level, the same diagrams arise for the quark bilinears with a gluon mass as would arise in the Curci-Ferrari theory [7] which is known both to be renormalizable and to recover QCD in the limit $\mu \rightarrow 0$. The use of the Curci-Ferrari formulation to regulate IR divergences arising during the calculation of IR finite gauge-invariant quantities is well established [8]. Beyond the one-loop level, processes in the Curci-Ferrari theory will receive contributions from additional diagrams compared to QCD, but at the one-loop level the inclusion of gluon and ghost masses suffices.

The IR logarithm $\log (\mu a)$ from lattice NRQCD then combines with the IR $\log$ arithm $\log (\mu / M)$ from continuum QCD side to yield the expected logarithmic $\log (M a)$-dependence for the matching coefficient.

For power divergences, such combinations are not possible, and hence these must match between QCD and NRQCD. The IR power divergences in the spin-independent are rather severe, going up to $\mathrm{O}\left(M^{3} / \mu^{3}\right)$, whereas the spin-dependent part is rather more mildly IR divergent, only up to $\mathrm{O}(M / \mu)$. We subtract the analytically known power divergences from the NRQCD integrands before performing the loop integrations. For the spin-dependent part this is enough to render it well-defined.

The spin-independent part, however, can also receive contributions from single-gluon exchange, which gives an $a^{2} \log (\mu a)$ IR divergence. This IR divergence cancels against an $a^{2} \log (\mu a)$ divergence in the sum of the box diagrams that arises where $\mathrm{O}\left(k^{2} a^{2}\right)$ lattice artifacts move the leading IR divergences to lower orders. The cancellation is ensured by the fact that these artifact divergences can only occur in diagrams involving the exchange of only temporal gluons, since the spatial gluon coupling carries an additional power of momentum. The artifact divergences thus 
survive the static limit and contribute to the Coulomb potential, which is $\mathrm{O}\left(a^{2}\right)$ improved for the Lüscher-Weisz action used here. Nevertheless, the presence of these artifact divergences at intermediate stages renders the calculation of the spin-independent part rather difficult; the calculation is now completed, and the results will be reported in the near future.

\section{Physical Impact}

The most immediate physical impact of the spin-dependent terms is to induce a hyperfine splitting (HFS) in heavy-quark mesons.

In the case of bottomonium, the leading contribution to the HFS from single-gluon exchange is $\sim c_{4}^{2}$; at the same order, the four-fermion interactions contribute $\sim\left(d_{1}-d_{2}\right)$. It is empirically found that the four-fermion contribution acts to reduce the discretization effects on the HFS; in addition, the larger value of the four-fermion coefficients found when including spin-dependent $\mathscr{O}\left(v^{6}\right)$ terms appears to partially compensate for the smaller HFS found with this action [9].

Taking both contributions together, the radiative improvement of the action has a significant impact on predictions, as it corrects the measured $1 \mathrm{~S} \mathrm{HFS} \sim 60 \mathrm{MeV}$ to $70 \mathrm{MeV}$, putting it into agreement with experiment [10]. Using the radiatively improved action, the HPQCD collaboration has also made the prediction of 35(3)(1) MeV for the 2S HFS [11], which agrees with the newest Belle results [12].

In the case of $B$ mesons, there are no four-fermion interactions that could contribute to the HFS, which receives its leading contribution $\sim c_{4}$ from single-gluon exchange. When including the radiative corrections to $c_{4}$, HPQCD finds good agreement with experiment for the HFS of the $B_{d}$ and $B_{s}$ systems. As a result a prediction for the $B_{c}$ HFS [13] becomes possible.

The impact of the Darwin term is much less noticeable: the bottomonium S-wave energy shift $\sim c_{2}$ will constitute only a very small effect except on very coarse lattices. However, since the masses of S-wave bottomonia are used to calibrate the lattice spacing, including the correction to the Darwin term will help to increase the overall accuracy of the lattice predictions.

\section{Conclusions}

The inclusion of radiative improvement into the lattice NRQCD action can be achieved by calculating quark form factors in BFG and matching the results between continuum QCD and lattice NRQCD. In addition, four-fermion terms incorporating the difference between QCD and NRQCD quark-antiquark scattering matrix elements need to be added to the NRQCD action.

What is found is that there is a noticeable difference between spin-dependent and spin-independent terms: the spin-dependent terms are only mildly affected by IR divergences and have significant physical impact, to the extent that the agreement of the theory with experiment depends on their radiative improvement.

The spin-independent terms are more IR divergent, making them more subtle to compute; at the same time, they have little impact on heavy-quark spectra.

Detailed numerical results for all coefficients will be given in a forthcoming publication [14].

Acknowledgements: We thank the DEISA Consortium, co-funded through the EU FP6 project RI-031513 and the FP7 project RI-222919, for support within the DEISA Extreme Computing Initiative. This work was supported by 
STFC under grants ST/G000581/1 and ST/H008861/1. GMvH was supported in part by the DFG in the SFB 1044. The calculations for this work were, in part, performed on the University of Cambridge HPCs as a component of the DiRAC facility jointly funded by STFC and the Large Facilities Capital Fund of BIS.

\section{References}

[1] G. P. Lepage, L. Magnea, C. Nakhleh, U. Magnea and K. Hornbostel, Phys. Rev. D 46 (1992) 4052 [hep-lat/9205007].

[2] T. C. Hammant, A. G. Hart, G. M. von Hippel, R. R. Horgan and C. J. Monahan, Phys. Rev. Lett. 107 (2011) 112002 [arXiv:1105.5309].

[3] T. C. Hammant, A. G. Hart, G. M. von Hippel, R. R. Horgan and C. J. Monahan, PoS(LATTICE 2012)154 [arXiv:1212.2849].

[4] B. S. DeWitt, Phys. Rev. 162 (1967) 1195; B. S. DeWitt, Phys. Rev. 162 (1967) 1239;

H. Kluberg-Stern and J. B. Zuber, Phys. Rev. D 12 (1975) 482; A. Rebhan, Nucl. Phys. B 288 (1987) 832.

[5] T. C. Hammant, R. R. Horgan, C. J. Monahan, A. G. Hart, E. H. Müller, A. Gray, K. Sivalingham and G. M. von Hippel, PoS(LATTICE 2010)043 [arXiv:1011.2696]; A. Hart, G. M. von Hippel, R. R. Horgan and E. H. Müller, Comput. Phys. Commun. 180 (2009) 2698 [arXiv:0904.0375].

[6] M. Lüscher and P. Weisz, Nucl. Phys. B 452 (1995) 213 [hep-lat/9504006].

[7] G. Curci and R. Ferrari, Nuovo Cim. A 32 (1976) 151; G. Curci and R. Ferrari, Nuovo Cim. A 35 (1976) 1; L. von Smekal, M. Ghiotti and A. G. Williams, Phys. Rev. D 78 (2008) 085016 [arXiv:0807.0480].

[8] G. Curci and E. d'Emilio, Phys. Lett. B 83 (1979) 199; I. Ojima, Z. Phys. C 13 (1982) 173; A. Blasi and N. Maggiore, Mod. Phys. Lett. A 11 (1996) 1665 [hep-th/9511068]; R. Browne and J. Gracey, Phys. Lett. B 540 (2002) 68 [hep-th/0206111]; K.-I. Kondo, K. Suzuki, H. Fukamachi, S. Nishino and T. Shinohara, arXiv:1209.3994.

[9] S. Meinel, Phys. Rev. D 82 (2010) 114502 [arXiv:1007.3966].

[10] R. J. Dowdall, PoS(LATTICE 2011)118 [arXiv:1111.0449].

[11] R. J. Dowdall et al. [HPQCD Collaboration], Phys. Rev. D 85 (2012) 054509 [arXiv:1110.6887].

[12] R. Mizuk et al. [Belle Collaboration], arXiv:1205.6351.

[13] R. J. Dowdall, C. T. H. Davies, T. C. Hammant and R. R. Horgan, arXiv:1207.5149.

[14] T. C. Hammant, A. G. Hart, G. M. von Hippel, R. R. Horgan and C. J. Monahan, in preparation. 